\title{
Evaluation of Occupational and Patient Dose in Challenging Hemodialysis Catheter Placement
}

\author{
Alireza Rasekhi, ${ }^{1,2}$ Amin Abolhasani Foroughi, ${ }^{1,2,}$ Banafsheh Zeinali-Rafsanjani, ${ }^{3,1}$ Mahdi \\ Saeedi-Moghadam, ${ }^{1}$ Fariba Zarei, ${ }^{1,2}$ and Masoume Nazeri ${ }^{4}$ \\ ${ }^{1}$ Medical Imaging Research Center, Shiraz University of Medical Sciences, Shiraz, Iran \\ ${ }^{2}$ Department of Radiology, Shiraz University of Medical Sciences, Shiraz, Iran \\ ${ }^{3}$ Nuclear Medicine and Molecular Imaging Research Center, Shiraz University of Medical Sciences, Shiraz, Iran \\ ${ }^{4}$ Department of Neurology, Clinical Neurology Research Center, Shiraz University of Medical Sciences, Shiraz, Iran \\ "Corresponding author: Amin Abolhasani Foroughi, Medical Imaging Research Center, Shiraz University of Medical Sciences, Shiraz, Iran. Tel: +98-7136281464, Fax: \\ +98-7136281507, E-mail: amin.a.foroughi@gmail.com
}

Received 2016 August 20; Revised 2017 January 16; Accepted 2017 April 16.

\begin{abstract}
Background: Catheter hemodialysis is the last choice for end stage kidney disease patients. Unfortunately, long-term catheterization can lead to problems that make re-catheterization challenging. Sometimes the physician has to try catheterization through several veins and stays close to the patient during the procedure that may lead to a high radiation dose exposure to the radiologist, so that some radiologists refuse to admit such patients.

Objectives: The objective of this study was an assessment of the radiation dose of radiologists and patients during the placement of double lumen catheter in difficult cases.

Patients and Methods: The thermoluminescent dosimeter (TLD) was used to measure the delivered radiation dose to physicians. During the procedure, a package of dosimeter was placed on the thyroid shield and another on the chest, under the apron in order to calculate the occupational effective dose. Moreover, patient information and parameters of the procedure were recorded in order to estimate the patient dose during the procedure.

Results: The mean effective dose provided by TLDs, dose/procedure, and the annual dose of physicians resulted from this procedure were $30.38 \mu \mathrm{Sv}, 0.02 \mu \mathrm{Sv} /$ procedure, and $350.20 \mu \mathrm{Sv} / \mathrm{y}$, respectively. The mean dose area product, dose, and calculated peak skin dose obtained in this study were $151.44 \mathrm{cGy} . \mathrm{cm}^{2}, 851.71 \mathrm{mGy}$, and $384.97 \mathrm{mGy}$, respectively.

Conclusion: The mean effective dose of physician per case is about $0.02 \mu \mathrm{Sv} /$ procedure which is much less than the radiation dose per other procedures. Briefly, it can conclude that double lumen replacement does not deliver an unacceptable radiation dose to the physician and even the radiation dose resulted from these cases are much less than the other procedures.
\end{abstract}

Keywords: Challenging Catheterization, Interventional Radiology, Radiation Dose

\section{Background}

The last choice for the end-stage kidney disease patients is catheter hemodialysis. In these cases, when the patient needs an immediate hemodialysis, a double lumen catheter should be placed through internal or external jugular veins. Unfortunately, long-term catheterization can lead to venous thrombosis, obstruction, and infection (1). As a result of these difficulties following catheterization, the catheter should be replaced, which gradually causes difficult accessibility to an appropriate place (2). In these cases, there is no standard method to choose the second most proper vein for catheterization. The available options are the subclavian vein, femoral vein, hepatic vein, trans-lumbar inferior vena cava, and collateral neck and chest veins (3).

When the blood flow rate through the catheter is more than $300 \mathrm{ml} / \mathrm{min}$, it has good functionality (4). Using this criterion, $87 \%$ of catheters lose their functionality at least once during their lives (5). Severe poor functionality that leads to catheter replacement occurs in $16 \%$ of catheters depending on the type of catheter (6).

Sometimes the physician has to try catheterization through several veins and stays close to the patient during the procedure which can take a lot of time in comparison to normal or other procedures. It is rational that more radiation doses are delivered to the radiologist during these complicated procedures. Previous dosimetric studies on radiation dose to physicians during interventional radiology had demonstrated that if radiologists do not use suitable radiation protective devices, their radiation dose could exceed the annual dose limit (7). There are also some reports of the occurrence of deterministic effects in interventionists (8-10).

According to the aforementioned issues, some radiol- 
ogists may refuse to accept such patients for intervention. Among the community of radiologists, considering that this is the last choice for the patient to live, the dutiful ones agree to perform the procedure in order to give a chance to the patient for living.

\section{Objectives}

Consequently, the objective of this study was an assessment of the radiation dose to radiologists during the placement of double lumen catheter in challenging cases, in order to estimate their annual radiation dose. Accordingly, it could be proved whether these kinds of catheterization deliver an unacceptable dose to the interventionist. In case the radiation dose to the physician does not exceed the annual dose, their point of view may change, and they could admit more patients in this situation. Therefore, more patients have the chance to live longer, which is very valuable for patients and their family. In addition, some other information such as dose area product (DAP) $\left(\mathrm{cGy} . \mathrm{cm}^{2}\right)$, dose (mGy), time (minute) of irradiation was recorded to calculate the dose to patients as well. Finally, the radiation dose to physicians and patients could be compared.

\section{Patients and Methods}

\subsection{Occupational Dose Measurement and Calculation}

The thermoluminescent dosimeter (TLD-100), which is a routine dosimeter for dose measurement in clinic, was used to measure the delivered radiation dose to physicians. The TLD dosimeters were packed in a triplex. During the double lumen procedure in complicated cases, a triplex package was placed on the thyroid shield and another on the chest under the apron (Figure 1). Since the radiologists' hands could had been exposed to primary radiation, the dose to the hands was measured by a ring dosimeter containing two TLDs. The TLD packs were worn by radiologists only for the challenging double lumen cases. The TLD dosimeters were used for a month by two radiologists who admitted these patients in the department of general angiography, Shiraz Nemazee hospital.

The results of TLDs were corrected for operational quantity HP (0.07) and HP (10) (1). Using the Nilklason algorithm, the effective dose was calculated as below.

$E=0.02(H O-H U)+H U$

Ho and HU are the results of TLDs (air kerma) on the thyroid shield and under the apron, respectively. Some studies declared that the effective doses which were estimated by this algorithm are more accurate and more agree with the international commission of radiation protection

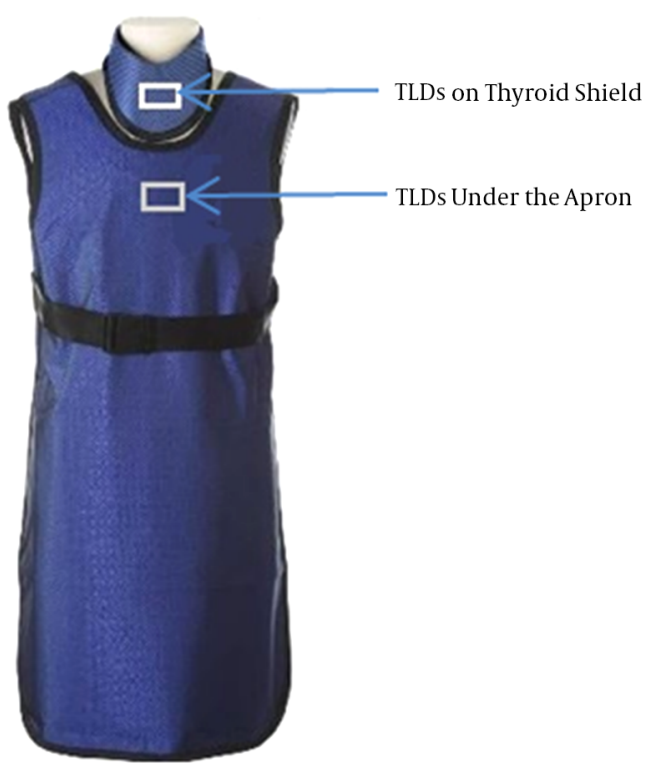

Figure 1. Locations of thermoluminescent dosimeters (TLD) that were used during the procedure

(ICRP 75). Moreover, this algorithm can provide a good estimation just by using two data points (under the apron and on the thyroid shield), while the others need more data points, which mean more TLDs, more cost, more time to prepare them and also more difficulties for the physicians who want to use the dosimeters (2-5).

\subsection{Patient Dose Measurement and Calculation}

During the procedures, patient information including age, height and weight and also parameters of the procedure including DAP, cumulative dose and irradiation time, which could be provided by the angiography machine (Siemens, Model No: 3800351), were recorded in order to provide a good estimation of patient dose during the procedure. According to a guideline for patient radiation dose management, the peak skin dose (PSD) could be calculated using DAP (6).

$$
P S D(m G y)=249+5.2 \times D A P\left(G y . \mathrm{cm}^{2}\right)
$$

\section{Results}

During a month, 14 patients who had previous experience of this procedure for two or more times were referred for double lumen catheter placement. The detail of patient information is demonstrated in Table 1.

The procedure information, including DAP $\left(\mathrm{cGy} . \mathrm{cm}^{2}\right)$, dose (mGy), and irradiation time (minute) were recorded 
Table 1. Characteristics of Patients Who Were Referred for Double Lumen Replacement

\begin{tabular}{lccc}
\hline Patient & Age & Height & Weight \\
\hline $\mathbf{1}$ & 25 & 168 & 70 \\
\hline $\mathbf{2}$ & 35 & 171 & 75 \\
\hline $\mathbf{3}$ & 32 & 152 & 58 \\
\hline $\mathbf{4}$ & 55 & 161 & 65 \\
\hline $\mathbf{5}$ & 62 & 150 & 78 \\
\hline $\mathbf{6}$ & 40 & 168 & 63 \\
\hline $\mathbf{7}$ & 35 & 174 & 62 \\
\hline $\mathbf{8}$ & 29 & 170 & 65 \\
\hline $\mathbf{9}$ & 50 & 185 & 96 \\
\hline 10 & 41 & 165 & 52 \\
\hline 11 & 40 & 165 & 70 \\
\hline 12 & 37 & 163 & 63 \\
\hline 13 & 42 & 160 & 65 \\
\hline 14 & 44 & 160 & 58 \\
\hline Average & 40.5 & 165.14 & 10.35 \\
\hline SD & 9.62 & 8.55 & \\
\hline
\end{tabular}

for each patient and the mean values were calculated. The PSD (mGy) was also estimated using Equation 2 (Table 2).

The mean effective dose provided by TLD readings and estimated using Nilklason algorithm, dose/procedure, and the annual dose to physicians resulted from this procedure were $30.38 \mu \mathrm{Sv}, 0.02 \mu \mathrm{Sv} /$ procedure, and $350.20 \mu \mathrm{Sv} / \mathrm{y}$, respectively. The mean cumulative dose of hands and its annual dose were also $1.34 \mathrm{mSv}$, and $16.08 \mathrm{mSv} / \mathrm{y}$, respectively.

\section{Discussion}

Since there was a belief that during double lumen placement, radiation dose to physicians could be so high and hazardous, radiologists may refuse to admit these kind of patients. This belief comes from the fact that in most cases they should try different veins to find the best way for catheterization, and most of the veins have endoluminal chronic thrombosis. Therefore, this procedure may be time-consuming, and the physician should stay with the patients and near the $\mathrm{x}$-ray tube during the procedure. Accordingly, effective doses to physicians were measured using TLD dosimeters and Nilklason algorithm. The radiation dose to patients was also assessed by recording the DAP and dose values provided by the $\mathrm{x}$-ray machine. Finally, PSD was calculated using DAP value provided by the x-ray machine.

According to Table 1, most of the patients referred for hemodialysis catheter placement were middle-aged peo- ple of medium height and weight. The exposure time in this procedure can be so varied from a minute to half an hour. In our center, during the period of study, the mean exposure time was 8.6 minutes and the maximum exposure time was 30 minutes. Although the extreme time was only half an hour, it led to delivering a high DAP and dose to the patients. The mean DAP (151.44 cGy.cm²), dose (851.71 mGy), and calculated PSD (384.97 mGy) obtained in this study (Table 2) were comparable to the values of a study conducted by Storm et al. (7), in which the obtained maximum dose and PSD values were $815 \mathrm{mGy}$, and 489 mGy, respectively. The maximum values of dose and PSD were taken into account since our study was performed for cases who had difficult accessibility and needed more irradiation time. The mean and max DAP values that were recorded in this study were less than the study performed by Storm et al. It could be attributed to the lower tube loading and beam output, collimation as well as the focus to skin distance, that is so variable during the procedure (8).

For a single procedure, the peak skin dose to the patient in the worst situation is about $1.5 \mathrm{~Gy}$, in which no observable effect happens. It should be noted that this procedure may be repeated for the patient so that the cumulative dose would be several times higher than the mean dose in some cases. The threshold radiation dose for skin deterministic effect is $2 \mathrm{~Gy}$ (9). Therefore, by just performing the test for the second time in sophisticated cases that need more time, it may result in skin erythema or even epilation. If the PSD reaches $15 \mathrm{~Gy}$ as a result of repetition, it should be reported to the radiation safety officer (RSO) and medical director (10). For more clarification, it could be said that the mean effective dose to the patients was $851.71 \mathrm{mSv}$, which is almost equal to 42 abdominal-pelvic computed tomographies (CT) with and without contrast (20 mSv) (11).

The mean effective dose to physicians per case is about $0.02 \mu \mathrm{Sv} /$ procedure (0.002 - $0.04 \mu \mathrm{Sv} /$ procedure) which is much less than the radiation dose per other procedures such as percutaneous nephrolithotomy (1.7-56 $\mu \mathrm{Sv})$, vertebroplasty (0.1 - $101 \mu \mathrm{Sv})$, orthopedic extremity nailing (2.5 - $88 \mu \mathrm{Sv})$, biliary tract procedure $(2.0$ - $46 \mu \mathrm{Sv})$, transjugular intrahepatic portosystemic shunt creation (2.5 - 74 $\mu \mathrm{Sv})$, head/neck endovascular therapeutic procedures (1.8 - 53 $\mu \mathrm{Sv})$, and endoscopic retrograde cholangiopancreatography $(0.2-49 \mu \mathrm{Sv})(12)$.

The calculated effective dose to the physician was 30.38 $\mu \mathrm{Sv}$ in one month for 14 cases, which is almost equal to 0.3 simple chest radiography ( $0.1 \mathrm{mSv} /$ procedure) (11). The annual dose to radiologists was $350.20 \mu \mathrm{Sv} / \mathrm{y}$ (0.35 mSv/y) which is much less than the occupational dose limit (20 $\mathrm{mSv} / \mathrm{y})(13)$.

According to dose guidance value provided by ICRP, the 
Table 2. Mean Value of DAP, Dose, Irradiation Time and PSD Compared to a Similar Study

\begin{tabular}{lcc}
\hline Procedure Information & Value & $\begin{array}{c}\text { Storm et al. Study (7) } \\
\text { Mean (Min - Max) Geometric Mean }\end{array}$ \\
\hline Mean DAP, cGy.cm2 & Mean (Min - Max) Geometric Mean & $485(32-10660) 512$ \\
Mean Dose, mGy & $151.44(10-556) 66.22$ & $43(2-815) 44$ \\
Mean Time, minute & $851.71(182-1800.20) 851.71$ & $2.7(0.3-24.6) 2.7$ \\
Mean PSD, mGy & $8.60(1.32-30) 5$ & $20(2-489) 22$ \\
\hline
\end{tabular}

Abbreviations: DAP, dose area product; PSD, peak skin dose.

dose limit of hands and feet is $500 \mathrm{mSv}$, which means that radiation doses less than this value do not lead to deterministic radiation-induced effects (13). $500 \mathrm{mSv}$ is much higher than the calculated annual radiation dose of hands $(16.08 \mathrm{mSv})$ in this procedure. Therefore, because the delivered radiation dose to hands was less than the dose limit, it can be concluded that performing these challenging cases does not cause any damage to hands provided that the interventionist considers a rational radiation protection.

We live in a world that naturally we get $3 \mathrm{mSv} / \mathrm{y}$ from background radiation, in which $2 \mathrm{mSv} / \mathrm{y}$ of this radiation dose comes from radon gas that accumulates in our house, and we breathe it. Our natural radiation dose increases about $0.03 \mathrm{mSv}$ during a long trip flight as a result of cosmic radiation (13). In this situation, $0.35 \mathrm{mSv} / \mathrm{y}$ which is approximately 10 times less than the background radiation dose, is not high enough to make physicians refuse to admit those patients who may die in a few days without dialysis. Performing this study may be more harmful in terms of radiation-induced side effects for the patients in comparison to the interventionist, but since it is the only life chance for these patients, it is completely justified to perform this procedure for the patient. Briefly, it can conclude that double lumen replacement does not deliver an unacceptable radiation dose to the physician and even the radiation dose resulted from these cases is much less than other procedures.

\section{References}

1. I. C. o. R. P. I. P. . Recommendations of the International Commission on Radiological Protection. Ann ICRP. 2007;103(37):1-332.
2. Padovani R, Foti C, Malisan MR. Staff dosimetry protocols in interventional radiology. Radiat Prot Dosimetry. 2001;94(1-2):193-6. [PubMed: 11487835].

3. Schultz FW, Zoetelief J. Estimating effective dose for a cardiac catheterisation procedure with single or double personal dosemeters. Radiat Prot Dosimetry. 2006;118(2):196-204. doi: 10.1093/rpd/ncl018. [PubMed: 16517567].

4. I. C. o. R. Protection. . General principles for the radiation protection of workers. ICRP Publication 75, Oxford: Pergamon Press,; 1997.

5. Jarvinen H, Buls N, Clerinx P, Jansen J, Miljanic S, Nikodemova D, et al. Overview of double dosimetry procedures for the determination of the effective dose to the interventional radiology staff. Radiat Prot Dosimetry. 2008;129(1-3):333-9. doi: 10.1093/rpd/ncn082. [PubMed: 18483007].

6. Stecker MS, Balter S, Towbin RB, Miller DL, Vano E, Bartal G, et al. Guidelines for patient radiation dose management. J Vasc Interv Radiol. 2009;20(7 Suppl):S263-73. doi: 10.1016/j.jvir.2009.04.037. [PubMed: 19560006].

7. Storm ES, Miller DL, Hoover LJ, Georgia JD, Bivens T. Radiation doses from venous access procedures. Radiology. 2006;238(3):1044-50. doi: 10.1148/radiol.2382042070. [PubMed: 16424240].

8. Akinlade BI, Farai IP, Okunade AA. Survey of dose area product received by patients undergoing common radiological examinations in four centers in Nigeria. J Appl Clin Med Phys. 2012;13(4):3712. doi: 10.1120/jacmp.v13i4.3712. [PubMed: 22766942].

9. Allisy-Roberts P, Williams JR. Farr's physics for medical imaging. Elsevier Health Sciences; 2007.

10. Arbique GM, Guild JB, Chason DP, Anderson J. The fluoroscopic sentinel event: What to Do. J Am Osteopath Coll Radiol. 2014;3:8-30.

11. American College of Radiology and Radiological Society of North America. . Radiation Dose in X-Ray and CT Exams. Available: Radiation Dose in X-Ray and CT Exams; 2016.

12. Kim KP, Miller DL, Berrington de Gonzalez A, Balter S, Kleinerman RA, Ostroumova E, et al. Occupational radiation doses to operators performing fluoroscopically-guided procedures. Health Phys. 2012;103(1):80-99. doi: 10.1097/HP.ob013e31824dae76. [PubMed: 22647920].

13. Radiation emergency medical measurement . International commis sion on radiological protection (ICRP), guidance for occupational exposure 2016. Available from: https://www.remm.nlm.gov/ICRP_ guidelines.htm. 